\title{
Excesos y alternativas de la salud mental en atención primaria
}

\section{Excessos e alternativas da saúde mental na atenção primária \\ Excesses and alternatives in mental health in primary care}

Alberto Ortiz Lobo. PhD, psiquiatra. Centro Salud Mental Salamanca, Hospital Universitario La Princesa. Madrid, España. lobortiza@gmail.com Jorge Bernstein, Presidente de las Comisiones de Salud Mental y Prevención Cuaternaria (FAMFyG). Presidente del Capítulo de Salud Mental en APS de la Asociación de Psiquiatras Argentinos. Buenos Aires, Argentina. jorge.bernstein@gmail.com (Autor correspondiente)

\section{Resumen}

La extraordinaria expansión de la psiquiatría y la psicología está propiciando el tratamiento de personas sanas que acaban siendo etiquetadas como trastornos mentales. El reduccionismo biológico del modelo médico centrado en los síntomas favorece la transformación de problemas sociales en conflictos individuales y la exposición de los ciudadanos a los efectos adversos de tratamientos excesivos e improcedentes. La prevención cuaternaria en salud mental contempla el empleo de narrativas y formulación de casos (más allá de la etiqueta diagnóstica), la indicación de no-tratamiento y un empleo de los psicofármacos prudente, que considere su deprescripción.

\section{Resumo}

A extraordinária expansão da psiquiatria e da psicologia está proporcionando o tratamento de pessoas saudáveis que acabam sendo rotuladas como transtornos mentais. 0 reducionismo biológico do modelo médico centrado em sintomas favorece a transformação de problemas sociais em conflitos individuais e a exposição dos cidadãos aos efeitos adversos de tratamentos excessivos e inadequados. A prevenção quaternária em saúde mental contempla o uso de narrativas e formulação de casos (para além do rótulo diagnóstico), a indicação de não-tratamento e um uso prudente de drogas psicoativas, que leve em consideração sua desprescrição.

\section{Abstract}

The extraordinary expansion of psychiatry and psychology is leading the treatment of healthy people who end up being labelled as mental disorders. The biological reductionism of the medical model centred on the symptoms favours both the transformation of social problems in individual conflicts and the exposure of citizens to the adverse effects of excessive and inappropriate treatments. Quaternary prevention in mental health contemplates the use of narratives and case formulation (beyond the diagnostic label), the indication of non-treatment, and prudent use of psychoactive drugs, taking into account its deprescription.
Palabras clave:

Salud Mental

Efectos adversos Enfermedad latrogénica Medicalización

Prevención Cuaternaria

\section{Palavras-chave:}

Saúde Menta

Efeitos adversos Doença latrogênica Medicalização Prevenção Quaternária

\section{Keywords:}

Mental Health

Adverse effects latrogenic Disease Medicalization Quaternary Prevention 


\section{Introducción}

La salud mental es un campo que se presta especialmente a que se dañe a los pacientes por lo que la prevención cuaternaria es una actividad crucial. La dificultad de acotar y conceptualizar los problemas mentales y la falta de tratamientos precisos y ajustados, entre otras particularidades, propicia una laxitud diagnóstica y terapéutica que pone en riesgo a la población (Tabla 1). El perjuicio que se puede infligir a los pacientes está presente en todas las actividades de la salud mental: prevención primaria, cribaje, diagnóstico, tratamiento y rehabilitación, por lo que es importante conocer qué excesos se cometen y qué alternativas podemos manejar en cada una de los procesos de atención. ${ }^{1}$ En personas con trastornos mentales graves el intervencionismo del sistema psiquiátrico y los internamientos pueden producir estigmatización y efectos adversos que marquen y dificulten la vida, pero en atención primaria se dańa principalmente a través del sobrediagnóstico y sobretratamiento de aquellos que consultan por sufrimientos vinculados a la vida cotidiana y que no constituyen trastornos mentales ni precisan actividades preventivas o curativas.

\section{La psiquiatrización y psicologización de la vida}

El fenómeno de la medicalización por el que cada vez más condiciones pasan a ser objeto de estudio y tratamiento en medicina, alcanza unas proporciones extraordinarias en el terreno de la salud mental. Muchas personas acuden a consulta con la expectativa de recibir un tratamiento para el malestar que se produce ante el afrontamiento de la vida cotidiana. En España, una cuarta parte de los pacientes que acuden a un servicio de salud mental derivados por su médico de cabecera no presentan un trastorno mental diagnosticable según criterios CIE-10 y la mitad de ellos ya tiene prescrito un psicofármaco. ${ }^{2}$ El tratamiento con medicinas o psicoterapia de los sentimientos desagradables (tristeza, angustia, frustración, impotencia, soledad, odio...) que aparecen en el contexto de una situación vital estresante como una respuesta adaptativa, legítima y proporcionada, expone a personas sanas a los daños que se derivan de intervenciones y diagnósticos sin un claro beneficio.

Las actividades de cribaje también constituyen un peligro potencial de perjuicio como en los casos de depresión ${ }^{3,4}$ o de Alzheimer, donde no existe tratamiento que lo justifique. La clave estaría en saber si ante el problema humano que tenemos delante vamos a obtener mejores resultados considerándolo una enfermedad a tratar que si no fuera visto como tal. Los protocolos de intervención no están bien definidos y no hay una tecnología que permita una verdadera objetivación del problema.

Tabla 1. Algunos factores que favorecen los excesos en salud mental.

\section{DE LA DISCIPLINA}

\begin{tabular}{l} 
Falta de una conceptualización definitiva de los problemas mentales \\
Desconocimiento final de las causas, generación y desarrollos de los problemas mentales. \\
Diversidad de teorías y tratamientos, a veces antagónicos entre sí. \\
Imposibilidad de trazar fronteras entre lo "normal" y lo "patológico". \\
Modelo hegemónico actual biomédico \\
Centrado en los síntomas y el individuo. \\
Desconsiderando el contexto, la Historia, los factores socioculturales, etc. \\
Muy influenciado por intereses comerciales y financieros. \\
Amparado en un autoritarismo pseudocientífico (cuya fundamentación neuroquímica no está probada). \\
Incapaz de aprehender lo humano (cultura, valores, significados, etc.) con sus herramientas positivistas. \\
\hline La constitución de la salud como un bien de consumo. \\
El poder de la industria farmacéutica en crear y expandir enfermedades mentales. \\
La consagración del individualismo (frente al abordaje colectivo de las conflictos sociales). \\
La exposición del sujeto, liberado de los anclajes tradicionales, a los riesgos de vivir y elegir (y la necesidad de expertos "psi" que los filtren y gestionen). \\
Tendencia a filiar asuntos morales, contradicciones sociales y deseos y frustraciones personales como problemas mentales. \\
\hline Conflictos intelectuales (incapacidad de considerar la disciplina y su tarea con cierto escepticismo, adhesión incondicional a una perspectiva y desprecio por el resto). \\
Conflictos personales (paternalismo, vanidad, exceso de compasión, omnipotencia, incapacidad para decepcionar, etc.).
\end{tabular}

Fuente: elaborado por los autores. 
Muchas de las consultas que realizan los pacientes tienen que ver con sufrimientos derivados de conflictos laborales, económicos, sociales... y en la medida que medicalizamos estas demandas, contribuimos a enmarcar en lo psicológico/biológico y en lo íntimo de una relación profesional-paciente, asuntos que son de orden ético y de ámbito público. Otras veces, en el manejo de estas demandas, ya sea en atención primaria o en salud mental, lo que estamos haciendo además es propugnar que nuestro paciente se adapte de forma personal (comprometiéndole a mejorar a él con nuestra intervención sanitaria), cuando lo que hay detrás son situaciones sociales injustas que exigen un análisis y una solución colectivas. ${ }^{5}$ En cualquier caso, corremos el peligro de favorecer un reduccionismo psicológico o biológico de fenómenos y realidades que son mucho más complejas y de empañar otras perspectivas que explican mejor y de forma más global el sufrimiento de nuestro paciente. ${ }^{6}$

La extensión del tratamiento en salud mental de forma indiscriminada a sufrimientos que no se pueden filiar como trastornos mentales conduce a una saturación de los dispositivos asistenciales y, consecuentemente, a una limitación de las prestaciones. La ley de cuidados inversos sostiene que el paciente recibe cuidados en proporción inversa a su necesidad. ${ }^{7}$ Con esta dinámica, se favorece que nuestras consultas se saturen de malestares para los que no hay una respuesta técnica sanitaria clara ni eficaz y que los trastornos mentales graves se pierdan o reciban una atención limitada. En este contexto, se está produciendo un aumento de la demanda para terapias psicológicas que precisan de un terapeuta que se convierta en un guía que aconseje al paciente sobre las decisiones de su vida, como si hubiera una forma de vivirla basada en conocimientos científicos.

\section{Consecuencias negativas del uso de etiquetas diagnósticas}

La nosología en psiquiatría y salud mental es un asunto muy controvertido. La extraordinaria variabilidad en número y contenido de las categorías diagnósticas solo en el último medio siglo da cuenta de su provisionalidad. Asimismo, algunos diagnósticos se van poniendo de moda por temporadas, ${ }^{8}$ como ha sucedido con la depresión desde los años 90 y más recientemente, el trastorno bipolar o el trastorno por déficit de atención e hiperactividad. Estas variaciones constatan que los diagnósticos no tienen un carácter sustantivo, de verdad inmutable, son construcciones cuya vigencia depende del momento histórico, de qué tipo de profesionales ostenta el poder de la disciplina y de los intereses económicos y sociales que hay detrás.

La elaboración de las sucesivas clasificaciones de las enfermedades mentales DSM y CIE ha producido, entre otros, dos fenómenos: la inflación del número de categorías diagnósticas y la reducción del umbral de los criterios por los que alguien es diagnosticado. Esto se ha traducido en un incremento extraordinario de la prevalencia de los trastornos mentales y así, según el DSM-IV, la mitad de la población podrá ser diagnosticada de al menos un trastorno mental a lo largo de su vida, ${ }^{9}$ para regocijo de la industria farmacéutica y muchos profesionales de la salud mental. Cabe esperar que esta prevalencia aumente con el DSM-5, que con decisiones como eliminar el duelo como criterio de exclusión en el diagnóstico de depresión, apunta a medicalizar más condiciones y personas. La creencia de que las categorías diagnósticas son discretas y arraigadas en una anormalidad refleja un modelo de enfermedad en el que los trastornos mentales son clases naturales. Las etiquetas diagnósticas no captan la realidad en forma de síntomas sino que la interpretan. Tienen una capacidad de cosificación extraordinaria y parecería que pueden definir y concretar la esencia de un sujeto. Cuando se dice de un paciente que tiene un trastorno de personalidad o que es un depresivo, se transforman las interpretaciones subjetivas de los síntomas que realiza el profesional en etiquetas que deshumanizan los problemas, restringen nuestra comprensión del individuo y nos inducen una visión sesgada en la que vamos a resaltar todo aquello que se ajuste al diagnóstico.

Tampoco hay que perder de vista que las clasificaciones de las enfermedades mentales se diseñan por especialistas hospitalarios de países occidentales. Estos suelen desconocer el tipo de demandas que se producen en atención primaria y, yendo más allá, su perspectiva no tiene nada que ver con la cultura de los países en desarrollo, donde la conceptualización de los problemas mentales y su abordaje (muchas veces a través de agentes no especializados) puede ser muy distinta. Incluso se ha afirmado que "la investigación indica que los pacientes con trastornos depresivos tienen diferente etiología, fisiopatología y evolución natural que los enfermos psiquiátricos hospitalizados. ${ }^{10}$ Esta colonización ideológica de las sociedades económicamente dominantes que desplaza las formas tradicionales y locales de evaluar y experimentar el sufrimiento psíquico no ha sido necesariamente positiva. ${ }^{11}$

Dentro de esta perspectiva positivista y operativa se está desarrollando la biometría en salud mental que promueve el uso de escalas para cribar y certificar diagnósticos o controlar seguimientos. Se toman estos resultados como si fueran una verdad objetiva, desplazando el juicio clínico, sin embargo la validación de las escalas se hace comparando sus resultados con el método patrón: la misma entrevista clínica. Una medición "objetiva” termina reemplazando el diálogo terapéutico entre personas. 


\section{Los daños de establecer una relación terapéutica}

Cuando indicamos un tratamiento a un paciente, implícitamente le estamos trasmitiendo muchos mensajes que pueden ser potencialmente perjudiciales. Lo que estamos haciendo es interpretar las características personales del paciente, su malestar o su relato vital en el marco de un sistema de creencias orientado a la patología. De esta manera, se le comunica de forma implícita que su problema no mejorará o incluso empeorará si no realiza el tratamiento y, si este se interrumpe de forma anticipada (lo que sucede en numerosas ocasiones), no habrá mejorado lo que estaba previsto o corre el peligro de ponerse mucho peor. Esta dependencia respecto de la intervención profesional tiende a empobrecer los aspectos no médicos que también pueden ser saludables y curativos del ambiente y tiende a reducir la capacidad psicológica del paciente para afrontar sus problemas. No se escapan a esta argumentación aquellos marcos basados en la psicología de la salud dirigidos a "potenciar" al sujeto o dotarlo de nuevos recursos. En estos casos el paciente sigue siendo alguien deficitario al que un "experto" le tiene que dar algo que él no alcanza ni puede conseguir por su cuenta.

El lenguaje psiquiátrico y psicoterapéutico también restringe la comprensión que realizan los propios pacientes de sus experiencias. El concepto de "normalidad" que tiene el paciente se estrecha y puede acabar codificando buena parte de sus conductas desde su condición psiquiátrica o psicológica. Esto puede limitar su sensación de autonomía y gobierno y favorece la necesidad de que sea el experto profesional el que gestione sus emociones y experiencias. ${ }^{12,13}$ La relación con un profesional favorece que el paciente se sitúe en un rol pasivo y enfermo ante los avatares de su vida y ya no tiene que contar sus problemas en su entorno lo que debilita el apoyo de las redes tradicionales de contención.

Existen además unos efectos adversos inherentes a la terapia. Cada modelo teórico tiene su particular sistema de entender e interpretar las conductas de los pacientes. A través de este sistema y conducido por un lenguaje particular, los terapeutas trasmiten que saben más que el propio paciente sobre sí mismo. Este sistema de creencias domina la conversación terapéutica y favorece que perspectivas alternativas que el paciente pueda considerar, queden relegadas porque sean contradictorias o estén fuera de la perspectiva particular del profesional. Las distintas visiones que aportan los modelos teóricos pueden ser muy útiles en la comprensión de los problemas del paciente, pero en ningún caso son la "verdad", ${ }^{14}$ algo a lo que es difícil que se sustraiga el paciente durante y después del tratamiento. Se ha señalado que los aspectos no específicos, por oposición a técnicas específicas, que dan cuenta de la mayor parte del cambio en psicoterapia. ${ }^{15}$ Los psicoterapeutas, o equipos, que solo manejan una técnica tienden a usar la misma en más ocasiones de las indicadas.

\section{Los tratamientos psicológicos y sus efectos adversos}

Las intervenciones psicoterapéuticas se han considerado tradicionalmente inocuas, puesto que en principio se trata de un intercambio dialéctico, sin más. Pero si reconocemos su potencial terapéutico, también tenemos que considerar su capacidad para dañar. Se ha calculado que entre un tres y un $15 \%$ de los pacientes empeoran tras realizar un tratamiento psicológico. ${ }^{16,17}$ Este perjuicio depende de muchas variables, como el contexto del paciente, la formación técnica del profesional y sus características personales, factores del proceso terapéutico (en especial, la capacidad de producirse una alianza terapéutica) y también variables que dependen de la especificidad de la terapia. Se han descrito intervenciones terapéuticas que resultan perjudiciales ya sea por sus características particulares o su ámbito de aplicación. ${ }^{18}$ Las más estudiadas, y a la vez muy comunes, son la realización de debriefing en supervivientes de acontecimientos traumáticos y el tratamiento psicológico del duelo. El debriefing se realiza con el propósito de reducir la incidencia del trastorno por estrés postraumático (TEPT) en ciudadanos que han sido víctimas de catástrofes naturales, accidentes, violencia, etc. Cuando esta intervención se llevan a cabo de forma generalizada, la evolución clínica de los pacientes analizados globalmente es peor y los pacientes tratados desarrollan más síntomas de TEPT que los que no recibieron debriefing. ${ }^{19}$ Es posible que tanto profesionales como pacientes estén satisfechos con el tratamiento, pero la realidad es que el pronóstico clínico es peor. Más frecuente en atención primaria es intervenir en el proceso de duelo de un paciente tras la pérdida de un ser querido. Igualmente, se ha demostrado que el tratamiento generalizado del duelo en jóvenes y adultos no aporta globalmente evoluciones clínicas positivas y muchos pacientes se encuentran peor. ${ }^{20,21}$ Así que no basta con ser bienintencionados y bondadosos y desear ayudar a nuestros pacientes, también tenemos que cuestionarnos nuestras prácticas porque pueden ser perjudiciales. La fantasía de inocuidad de los tratamientos psicológicos conlleva el riesgo de sobretratamiento. 


\section{latrogenia del tratamiento psicofarmacológico}

Cada vez hay más pruebas de que la posible eficacia de los psicofármacos no se debe a supuestos mecanismos bioquímicos específicos que se ajustan a un diagnóstico o a una sintomatología. Las pocas categorías de fármacos que se emplean en psiquiatría se utilizan de forma inespecífica en un gran abanico de trastornos. Parece que los psicofármacos no corrigen desequilibrios en los neurotransmisores sino que, al contrario, los provocan e inducen estados psicológicos que pueden resultar útiles de forma inespecífica en el tratamiento de ciertos síntomas. ${ }^{22,23}$ Este efecto difuso de los psicofármacos pone cada vez más en perspectiva su efectividad, y alerta sobre los dańos que pueden causar.

Se cuestiona la creencia de que los neurolépticos sean un tratamiento crónico imprescindible para las psicosis, ${ }^{24,25}$ se acumulan pruebas sobre las limitaciones que producen y se consideran una posible barrera para la recuperación de las personas que los toman. Hay datos que apoyan la idea de que parte de la cronicidad y de las recaídas se deben precisamente a estar en tratamiento con neurolépticos, porque pueden producir psicosis de rebote, por sensibilización, disfrenia tardía, discinesia tardía, y síntomas negativos, ${ }^{26-31}$ además de una reducción del volumen cerebral de los que los utilizan. ${ }^{32,33}$

Sobre las benzodiacepinas hay mucha literatura y sabemos que el efecto adverso más relevante del uso crónico, más allá de la dependencia, ${ }^{34}$ es el deterioro cognitivo. ${ }^{35}$ Respecto a los antidepresivos, además de la polémica teoría de su capacidad para cronificar la depresión, ${ }^{36}$ su eficacia per se ha sido muy cuestionada en los últimos ańos. Los últimos meta-análisis dan cuenta de que la mayoría de los beneficios de los antidepresivos pueden explicarse por el efecto placebo y solo alrededor del $20 \%$ de la varianza puede ser atribuible al fármaco. ${ }^{37-39}$ Comparados con el placebo, los antidepresivos no son más eficaces en las depresiones leves o moderadas y, en todo caso, se muestran superiores en el tratamiento de las depresiones más graves. ${ }^{40,41}$

Todo esto teniendo en cuenta que los datos sobre eficacia de los psicofármacos están sobreestimados y la información sobre sus efectos adversos minimizada porque la investigación y su difusión está mayoritariamente controlada por la industria farmacéutica. Este control, que perpetúa que las muertes por medicamentos ocupen la tercera causa en el mundo occidental, ha propiciado la extraordinaria expansión del mercado de los psicofármacos bajo la apariencia de que son muy seguros y eficaces. ${ }^{42}$

Los daños que producen los psicofármacos se multiplican en las poblaciones más vulnerables. Los ancianos tienen la particularidad de estar además polimedicados por lo que los efectos adversos son más numerosos e impredecibles. Es muy frecuente el uso de neurolépticos en personas mayores con deterioro cognitivo con el fin de sedarlos, contenerlos o controlar sus conductas, no de forma terapéutica. El uso de neurolépticos en demencia multiplica por dos la tasa de muerte y el empleo de estos o de antidepresivos en mayores incrementa extraordinariamente la morbilidad en forma de accidentes vasculares, mareos, caídas, fracturas, problemas metabólicos y convulsiones. ${ }^{43,44}$ Igualmente, el uso de neurolépticos en discapacitados intelectuales tienen el objetivo de sedar, no de tratar a los sujetos. Estas prescripciones van en contra de los intereses del paciente al que se expone a riesgos graves y cuya conducta se podría manejar alternativamente con intervenciones psicosociales eficaces. ${ }^{45,46}$ Los niños tampoco escapan a la descontrolada expansión de la psiquiatría y los tratamientos psicofarmacológicos que actúan en este caso en un cerebro en desarrollo. Se desconoce el efecto a largo plazo que tendrá el consumo de estimulantes y neurolépticos administrados en esta generación fundamentado en diagnósticos tan cuestionables como el trastorno por déficit de atención e hiperactividad o el trastorno bipolar infantil.

Las guías de prescripción clínica pueden no ser útiles en el proceso de decidir si tratar y con qué: el 90\% de los autores que participaron en tres de ellas de gran repercusión tenían lazos financieros con las empresas farmacéuticas cuyos productos aparecían recomendados y este conflicto de intereses no aparecía reflejado en la guía. ${ }^{47}$ En estas circunstancias y con todos estos datos controvertidos, la prescripción se convierte en una tarea compleja en la que debemos calibrar cuidadosamente el balance riesgo-beneficio.

\section{Alternativas para reducir los perjuicios en salud mental}

Los juicios clínicos en medicina están muy lejos de ser análisis objetivos de un conjunto de hechos eminentemente medibles tecnológicamente, pero en el caso de la psiquiatría en particular, el cuestionamiento a la perspectiva médica y el modelo de enfermedad ha de ser radical. El diagnóstico alcanza su máxima subjetividad en este campo donde no hay medidas objetivas proporcionadas por la tecnología y cuya expresión y comprensión psicopatológica está muy ligada a la cultura. ${ }^{48}$ La etiqueta diagnóstica oculta la singularidad del paciente, la compleja interacción de factores genéticos, psicológicos, sociales 
y ambientales de ese individuo concreto, sus valores, significados, expectativas y necesidades que están inmersos en la cultura como marco organizador. Por ello, frente al diagnóstico simplificador, las narrativas nos proporcionan el significado, contexto y perspectiva del sufrimiento del paciente, define cómo, por qué y de qué manera está enfermo. La formulación de casos en salud mental (Tabla 2) permite, frente a la etiqueta diagnóstica, organizar la información clínica y dar una respuesta más ajustada a la singularidad del paciente. ${ }^{49}$

Antes de iniciar un tratamiento psicológico o farmacológico tenemos que considerar la posibilidad de indicar no-tratamiento. Esta es la intervención paradigmática de la prevención cuaternaria en salud mental ya que evita la exposición del paciente a los efectos adversos de los tratamientos. Muchas de las personas sanas que están sufriendo de manera proporcionada y adaptativa ante un evento vital esperan recibir un tratamiento cuando lo prudente es esperar y ver. Por ello, la indicación de no-tratamiento ha de ser una intervención frecuente y fundamental en el día a día de las consultas de Atención Primaria y Salud Mental. En estos casos, lo más beneficioso para el paciente será protegerle de los efectos secundarios de un tratamiento improcedente y para ello, primero tenemos que escuchar y hacernos cargo del sufrimiento del paciente y luego, con él, desvincularlo de que sea patológico o de que precise una intervención sanitaria y contextualizarlo dentro de su historia vital y de salud..$^{50}$ Esta indicación de no-tratamiento precisa de nuestras habilidades de entrevista psicoterapéuticas para que el paciente se sienta bien atendido (Tabla 3). Es la espera vigilante que ha de respetarse, por ejemplo, en un duelo normal, antes de pautar antidepresivos o realizar intervenciones psicológicas. Si un sujeto afronta y resuelve un duelo sin la necesidad de tratamientos, se refuerza su capacidad y autonomía a la hora de enfrentar acontecimientos adversos y de gestionar los sentimientos que producen sufrimiento. Se certifica su salud, su fortaleza y su resiliencia. Sin embargo, si tratamos a una persona que experimenta un duelo normal, igualmente mejorará como el primero (los duelos normales se caracterizan por eso, porque son autolimitados), pero implícitamente se le están transmitiendo otros mensajes: el tratamiento certifica, sino una patología, sí la incapacidad de esa persona de salir adelante por sí misma, su vulnerabilidad y su necesidad de consultar con un experto sanitario siempre que experimente sentimientos que produzcan sufrimiento, aunque estos sean sanos, legítimos y adaptativos.

Tabla 2. Ejemplo de formulación de casos de salud mental en Atención Primaria
1. DIAGNÓSTICO CLÍNICO DESCRIPTIVO
Descripción de los síntomas, curso y grado de afectación funcional.

\section{DIAGNÓSTICO SITUACIONAL}
2.1 Factores desestabilizadores actuales
2.2 Factores protectores/vulnerabilidad desde las dimensiones bio-psico-social:
Antecedentes médicos generales
Antecedentes psiquiátricos
Factores familiares
Factores sociales (económicos, laborales, red social, ocio...)
Rasgos de personalidad y estilos de afrontamiento

\section{MODELO EXPLICATIVO DEL PACIENTE}

Significado de los síntomas, atribuciones etiológicas.

\section{EXPECTATIVAS Y DEMANDAS DEL PACIENTE}

\section{PLAN TERAPÉUTICO}

\subsection{Objetivos}

5.2. Plan

\section{DEVOLUCIÓN}

Explicación sencilla y razonada de lo que le sucede al paciente que procure incluir los síntomas clínicos, los factores biopsicosociales y que pueda integrar, al menos parcialmente, el modelo explicativo del paciente y que dé una respuesta realista a sus expectativas.

Fuente: Mata Ruiz et al. ${ }^{49}$ 
Tabla 3. Mapa psicoterapéutico de la indicación de no-tratamiento.

Fases Objetivos

Trabajos

\begin{tabular}{|c|c|c|}
\hline \multirow{3}{*}{ 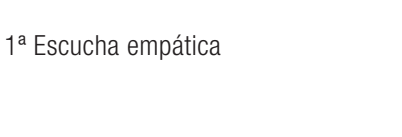 } & Enterarnos de lo que le sucede al paciente & Cuál es el problema \\
\hline & Que el paciente sepa que nos estamos enterando de lo que le sucede & Cómo se explica el problema \\
\hline & & Qué quiere (demanda) \\
\hline & & Qué papel nos otorga \\
\hline $2^{\mathrm{a}}$ Construcción de una versión inicial & Acordar con el paciente una versión inicial de forma conjunta & Emociones asociadas \\
\hline \multirow{3}{*}{$3^{\mathrm{a}}$ Deconstrucción } & Desvincular el problema que nos presenta el paciente de lo patológico y & \\
\hline & su solución del ámbito de lo sanitario & Del problema \\
\hline & & De cómo se lo explica \\
\hline \multirow{5}{*}{$4^{a}$ Resignificación } & Co-construir una nueva versión en que: & De la demanda \\
\hline & la problemática que plantea el paciente quede vinculada a su contexto & De la relación terapéutica \\
\hline & cotidiano saludable & Trabajo sobre las emociones \\
\hline & la carga emocional esté legitimada y normalizada & \\
\hline & el rol de enfermo cambie a uno más activo e independiente & \\
\hline $5^{\text {a }}$ Cierre & Despedirse & Condiciones del alta \\
\hline
\end{tabular}

Fuente: Ortiz Lobo et al. ${ }^{50}$

En este contexto de promoción comercial de los medicamentos que favorece la creación de unas expectativas irreales en pacientes y profesionales y un uso improcedente y excesivo de los mismos es necesario apelar a un uso juicioso de los psicofármacos. Es imprescindible una prescripción más estratégica, con una estrecha vigilancia de los efectos adversos, ser cauto y escéptico con las novedades, trabajar con los pacientes en una prescripción compartida y considerar el impacto de los psicofármacos a largo plazo. ${ }^{51}$ En esta línea de trabajo prudente, la deprescripción es otra actividad crucial. Muchas veces se mantienen de forma crónica antidepresivos, neurolépticos y benzodiacepinas no porque estén aportando un beneficio neto sino por la creencia de que "algo harán". Es verdad que, cuando se suprimen los psicofármacos algunos pacientes empeoran, pero en numerosas ocasiones esto es debido al síndrome de abstinencia que ha producido una prescripción prolongada y su retirada demasiado brusca.

\section{Conclusión}

Los intereses comerciales, corporativos y sociales y los conflictos intelectuales pueden pervertir la práctica de la salud mental y dañar a los ciudadanos. Las soluciones tecnológicas (proceso diagnóstico, administración de psicofármacos, psicoterapia) no son neutrales, están ligadas a unos valores. Solo con un uso reflejo, autocrítico y escéptico pueden hacerse explícitos estos valores y desde ahí podrán ser defendidos o rechazados.

\section{Referencias}

1. Ortiz Lobo A. Hacia una psiquiatría crítica. Madrid: Editorial Grupo 5; 2013

2. Ortiz Lobo A, González González R, Rodríguez Salvanés F. La derivación a salud mental de pacientes sin un trastorno psíquico diagnosticable. Aten Primaria. 2006;38(10):563-569. http://dx.doi.org/10.1157/13095928. PMid:17198609.

3. Thombs BD, Ziegelstein RC. Does depression screening improve depression outcomes in primary care? BMJ. 2014; 348:g1253. http://dx.doi.org/10.1136/bmj.g1253. PMid:24496211.

4. Gilbody S, House AO, Sheldon TA. Instrumentos de cribaje (screening) y detección de casos para la depresión. In: La Biblioteca Cochrane Plus [Internet]. Oxford: Update Software Ltd.; 2008. [última consulta el 2015 June 16]. Disponible en:

http://es.summaries.cochrane.org/cd002792/instrumentos-de-cribaje-screening-y-deteccion-de-casos-para-la-depresion\#sthash.tzlJquDm.dpuf. 
5. Rendueles G. Psiquiatrización de la ética - Ética de la psiquiatría: el idiota moral. In: Santander F, editor. Ética y praxis psiquiátrica. Madrid: AEN Colección Estudios; 2000

6. Jervis G. Manual crítico de psiquiatría. Barcelona: Anagrama; 1977

7. Hart JT. The inverse care law. Lancet. 1971;1(7696):405-412. http://dx.doi.org/10.1016/S0140-6736(71)92410-X. PMid:4100731.

8. Frances A. ¿Somos todos enfermos mentales? Buenos Aires: Ariel; 2014.

9. Kessler RC, Berglund P, Demler O, Jin R, Merikangas KR, Walters EE. Lifetime prevalence and age-of-onset distributions of DSM-IV disorders in the National Comorbidity Survey Replication. Arch Gen Psychiatry. 2005;62(6):593-602. http://dx.doi.org/10.1001/archpsyc.62.6.593. PMid:15939837.

10. Arroll B, Elley CR, Fishman T, Goodyear-Smith FA, Kenealy T, Blashki G, et al. Antidepresivos versus placebo para la depresión en la atención primaria. In: Biblioteca Cochrane Plus [Internet]. Oxford: Update Software Ltd.; 2009. [última consulta el 2015 June 16]. Disponible en: http://www.update-software.com.

11. Timimi S. Campaign to abolish psychiatric diagnosic systems such as ICD and DSM (CAPSID) [Internet]. Location: Publisher, year. [última consulta el 2015 June 16]. Disponible en: http://www.criticalpsychiatry.net/wp-content/uploads/2011/05/CAPSID12.pdf.

12. Boisvert CM, Faust D. latrogenic symptoms in psychotherapy. A theoretical exploration of the potential impact of labels, language, and belief systems. Am J Psychother. 2002;56(2):244-259. PMid:12125301.

13. Crown S. Contraindications and dangers of psychotherapy. Br J Psychiatry. 1983;143(5):436-441. http://dx.doi.org/10.1192/bjp.143.5.436. PMid:6640210.

14. Fernández Liria A, Rodríguez Vega B. La práctica de la psicoterapia. La construcción de narrativas terapéuticas. Bilbao: Desclée De Brower; 2001

15. Bracken P, Thomas P, Timimi S, Moldavsky D. La Psiquiatría más allá del paradigma actual. Acta Psiquiátr Psicol Am Lat. 2013;59(3):186-195.

16. Moos $\mathrm{RH}$. latrogenic effects of psychosocial interventions for substance use disorders: prevalence, predictors, prevention. Addiction. 2005;100(5):595-604. http://dx.doi.org/10.1111/j.1360-0443.2005.01073.x. PMid:15847616.

17. Mohr DC. Negative outcome in psychotherapy: A critical review. Clin Psychol Sci Pract. 1995;2(1):1-27. http://dx.doi.org/10.1111/j.1468-2850.1995.tb00022.x.

18. Lilienfeld SO. Psychological treatments that cause harm. Perspect Psychol Sci. 2007;2(1):53-70. http://dx.doi.org/10.1111/j.1745-6916.2007.00029.x.

19. Rose S, Bisson J, Churchill R, Wessely S. Psychological debriefing for preventing post traumatic stress disorder (PTSD). Cochrane Database Syst Rev. 2002;(2):CD000560. PMid:12076399.

20. Currier JM, Holland JM, Neimeyer RA. The effectiveness of bereavement interventions with children: a meta-analytic review of controlled outcome research. J Clin Child Adolesc Psychol. 2007;36(2):253-259. http://dx.doi.org/10.1080/15374410701279669. PMid:17484697.

21. Currier JM, Neimeyer RA, Berman JS. The effectiveness of psychotherapeutic interventions for bereaved persons: a comprehensive quantitative review. Psychol Bull. 2008;134(5):648-661. http://dx.doi.org/10.1037/0033-2909.134.5.648. PMid:18729566.

22. Moncrieff J, Cohen D. Rethinking models of psychotropic drug action. Psychother Psychosom. 2005;74(3):145-153. http://dx.doi.org/10.1159/000083999. PMid:15832065.

23. Healy D. The creation of psychopharmacology. Cambridge: Harvard University Press; 2002.

24. Bola JR, Mosher LR. At issue: predicting drug-free treatment response in acute psychosis from the Soteria project. Schizophr Bull. 2002;28(4):559575. http://dx.doi.org/10.1093/oxfordjournals.schbul.a006964. PMid:12795491.

25. Seikkula J, Aaltonen J, Alakare B, Haarakangas K, Keranen J, Lehtinen K. Five-year experience of first-episode nonaffective pychosis in opendialogue approach: Treatment principles, follow-up outcomes, and two case studies. Psychother Res. 2006;16(2):214-228. http://dx.doi.org/10.1080/10503300500268490.

26. Husa AP, Rannikko I, Moilanen J, Haapea M, Murray GK, Barnett J, et al. Lifetime use of antipsychotic medication and its relation to change of verbal learning and memory in midlife schizophrenia - An observational 9-year follow-up study. Schizophr Res. 2014;158(1-3):134-141. http://dx.doi.org/10.1016/j.schres.2014.06.035. PMid:25034761.

27. Frota LH. Partial agonists in the schizophrenia armamentarium. Tardive Dysphrenia: The newest challenge to the last generation atypical antipsychotics drugs? J Bras Psiquiatr. 2003;52(Suppl 1):14-24.

28. Fernandez HH, Trieschmann ME, Okun MS. Rebound psychosis: effect of discontinuation of antipsychotics in Parkinson's disease. Mov Disord. 2005;20(1):104-105. http://dx.doi.org/10.1002/mds.20260. PMid:15390047.

29. Margolese HC, Chouinard G, Beauclair L, Bélanger MC. Therapeutic tolerance and rebound psychosis during quetiapine maintenance monotherapy in patients with schizophrenia and schizoaffective disorder. J Clin Psychopharmacol. 2002;22(4):347-352. http://dx.doi.org/10.1097/00004714-200208000-00003. PMid:12172332.

30. Chouinard G, Chouinard VA. Atypical antipsychotics: CATIE study, drug-induced movement disorder and resulting iatrogenic psychiatric-like symptoms, supersensitivity rebound psychosis and withdrawal discontinuation syndromes. Psychother Psychosom. 2008;77(2):69-77. http://dx.doi.org/10.1159/000112883. PMid:18230939.

31. Moncrieff J. Does antipsychotic withdrawal provoke psychosis? Review of the literature on rapid onset psychosis (supersensitivity psychosis) and withdrawal-related relapse. Acta Psychiatr Scand. 2006 Jul;114(1):3-13. http://dx.doi.org/10.1111/j.1600-0447.2006.00787.x. PMid:16774655. 
32. Ho BC, Andreasen NC, Ziebell S, Pierson R, Magnotta V. Long-term antipsychotic treatment and brain volumes: a longitudinal study of firstepisode schizophrenia. Arch Gen Psychiatry. 2011;68(2):128-137. http://dx.doi.org/10.1001/archgenpsychiatry.2010.199. PMid:21300943.

33. Veijola J, Guo JY, Moilanen JS, Jääskeläinen E, Miettunen J, Kyllönen M, et al. Longitudinal changes in total brain volume in schizophrenia: relation to symptom severity, cognition and antipsychotic medication. PLoS One. 2014; 9(7):e101689. http://dx.doi.org/10.1371/journal.pone.0101689. PMid:25036617.

34. Ashton H. The diagnosis and management of benzodiazepine dependence. Curr Opin Psychiatry. 2005;18(3):249-255. http://dx.doi.org/10.1097/01.yco.0000165594.60434.84. PMid:16639148.

35. Billioti de Gage S, Moride Y, Ducruet T, Kurth T, Verdoux H, Tournier M, et al. Benzodiazepine use and risk of Alzheimer's disease: case-control study. BMJ. 2014; 349:g5205. http://dx.doi.org/10.1136/bmj.g5205. PMid:25208536.

36. Fava GA. Can long-term treatment with antidepressant drugs worsen the course of depression? J Clin Psychiatry. 2003;64(2):123-133. http://dx.doi.org/10.4088/JCP.v64n0204. PMid:12633120.

37. Turner EH, Matthews AM, Linardatos E, Tell RA, Rosenthal R. Selective publication of antidepressant trials and its influence on apparent efficacy. N Engl J Med. 2008;358(3):252-260. http://dx.doi.org/10.1056/NEJMsa065779. PMid:18199864.

38. Kirsch I, Deacon BJ, Huedo-Medina TB, Scoboria A, Moore TJ, Johnson BT. Initial severity and antidepressant benefits: a meta-analysis of data submitted to the Food and Drug Administration. PLOS Med. 2008;5(2):e45. http://dx.doi.org/10.1371/journal.pmed.0050045. PMid:18303940.

39. Pigott HE, Leventhal AM, Alter GS, Boren JJ. Efficacy and effectiveness of antidepressants: current status of research. Psychother Psychosom. 2010;79(5):267-279. http://dx.doi.org/10.1159/000318293. PMid:20616621.

40. Fournier JC, DeRubeis RJ, Hollon SD, Dimidjian S, Amsterdam JD, Shelton RC, et al. Antidepressant drug effects and depression severity: a patient-level meta-analysis. JAMA. 2010;303(1):47-53. http://dx.doi.org/10.1001/jama.2009.1943. PMid:20051569.

41. Barbui C, Cipriani A, Patel V, Ayuso-Mateos JL, van Ommeren M. Efficacy of antidepressants and benzodiazepines in minor depression: systematic review and meta-analysis. Br J Psychiatry. 2011;198(1):11-16. http://dx.doi.org/10.1192/bjp.bp.109.076448. PMid:21200071.

42. Gøtzsche P. Medicamentos que matan y crimen organizado. Barcelona: Ed. Los libros del lince; 2014.

43. Coupland C, Dhiman P, Morriss R, Arthur A, Barton G, Hippisley-Cox J. Antidepressant use and risk of adverse outcomes in older people: population based cohort study. BMJ. 2011;343. http://dx.doi.org/10.1136/bmj.d4551.

44. Morales BC. Síndrome metabólico y antipsicóticos de segunda generación. Rev Asoc Esp Neuropsiquiatr. 2011;31(2):303-320. http://dx.doi.org/10.4321/S0211-57352011000200009.

45. Wang PS, Schneeweiss S, Avorn J, Fischer MA, Mogun H, Solomon DH, et al. Risk of death in elderly users of conventional vs. atypical antipsychotic medications. N Engl J Med. 2005;353(22):2335-2341. http://dx.doi.org/10.1056/NEJMoa052827. PMid:16319382.

46. Ballard C, Hanney ML, Theodoulou M, Douglas S, McShane R, Kossakowski K, et al. The dementia antipsychotic withdrawal trial (DART-AD): long-term follow-up of a randomised placebo-controlled trial. Lancet Neurol. 2009;8(2):151-157. http://dx.doi.org/10.1016/S1474-4422(08)70295-3. PMid:19138567.

47. Cosgrove L, Bursztajn HJ, Krimsky S, Anaya M, Walker J. Conflicts of interest and disclosure in the American Psychiatric Association's Clinical Practice Guidelines. Psychother Psychosom. 2009;78(4):228-232. http://dx.doi.org/10.1159/000214444. PMid:19401623.

48. Launer J. Narrative based medicine: a narrative approach to mental health in general practice. BMJ. 1999;318(7176):117-119. http://dx.doi.org/10.1136/bmj.318.7176.117. PMid:9880290.

49. Mata Ruiz I, Ortiz Lobo A. Formulación de casos en salud mental: una guía de entrenamiento. In: Retolaza A. Trastornos mentales comunes: manual de orientación. Madrid: Asociación Española de Neuropsiquiatría; 2009. p. 195-213. Estudios 41.

50. Ortiz-Lobo A, Murcia-García L. La indicación de no-tratamiento: aspectos psicoterapéuticos. In: Retolaza A. Trastornos mentales comunes: manual de orientación. Madrid: Asociación Española de Neuropsiquiatría; 2009. p. 179-193. Estudios 41.

51. Schiff GD, Galanter WL, Duhig J, Lodolce AE, Koronkowski MJ, Lambert BL. Principles of conservative prescribing. Arch Intern Med. 2011;171(16):1433-1440. http://dx.doi.org/10.1001/archinternmed.2011.256. PMid:21670331. 